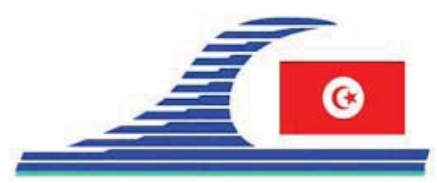

Conférence Méditerranéenne Côtière et Maritime EDITION 1, HAMMAMET, TUNISIE (2009)

Coastal and Maritime Mediterranean Conference

Disponible en ligne - http://www.paralia.fr-Available online

\title{
Morphodynamique des rides de sable dans le cas de sédiments homogènes ou hétérogènes en taille
}

\author{
Julie LEBUNETEL ${ }^{1}$, Armelle JARNO-DRUAUX ${ }^{2}$, François MARIN ${ }^{2}$
}

1. Cabinet ERAMM, « Le Thélème », 1503 route des Dolines, BP 42, 06901 Sophia Antipolis Cedex, France.

jl@eramm.fr

2. Laboratoire Ondes et Milieux Complexes (LOMC) FRE CNRS 3102, Université du Havre, 53 rue de Prony, BP 540, 76058 Le Havre, France.

armelle.jarno-druaux@univ-lehavre.fr ; francois.marin@univ-lehavre.fr

\section{Résumé :}

Ce travail à caractère expérimental vise à étudier la dynamique de formation des réseaux de rides de sable en canal à houle. Dans un premier temps, les expériences sont réalisées en sollicitant un fond sableux constitué de sédiments uniformes en taille avec des conditions de houles régulières. On étudiera l'évolution temporelle de ces structures à la fois d'un point de vue local (dimensions des rides en terme de longueur d'onde $L$, hauteur $h$, cambrure $h / L$ et section $S$ ) et d'un point de vue global en termes de statistique du réseau de rides présent sur l'ensemble de la veine d'essai $(6 m \times 0,35 \mathrm{~m})$. L'objectif est de caractériser précisément la croissance des rides et la dynamique du réseau afin d'accéder à la compréhension des processus intervenant au cours de la formation de ce type de structure. Dans un deuxième temps, les expériences sont réalisées avec un fond sédimentaire hétérogène en taille. L'influence de l'hétérogénéité des sédiments sur la croissance des rides est étudiée. Le tri sédimentaire en surface des rides est observé à l'aide de prélèvements réalisés au cours du temps dans la veine d'essai. Une technique expérimentale innovante a permis de caractériser le tri sédimentaire en profondeur pour les deux cas testés.

\section{Mots-clés :}

Rides de sable - Ecoulement oscillant - Tri sédimentaire - Canal à houle - Dynamique côtière

\section{Introduction}

L'étude de la dynamique sédimentaire en zone côtière fait partie intégrante des préoccupations actuelles liées à l'environnement. Sous certaines conditions de houle, des structures sédimentaires d'échelles spatio-temporelles variées se forment, résultant de l'interaction entre un écoulement oscillant et un milieu granulaire poreux. Les rides, souvent parallèles au rivage, sont des petites structures qui apparaissent sous forme de 
réseaux. Elles ont une influence significative sur la dissipation d'énergie de la houle ainsi que sur le transport de sédiments.

De nombreuses études ont déjà été réalisées concernant la formation de ce type de structure depuis les travaux pionniers de BAGNOLD (1946). Cependant les résultats des auteurs sur les dimensions à l'équilibre des rides diffèrent. La dispersion des résultats peut notamment être attribuée à la difficulté d'estimation des grandeurs caractéristiques des rides dans un réseau qui n'est jamais parfaitement régulier.

In situ, les fonds marins sont constitués de sédiments de tailles et formes variées. Ces paramètres influencent notamment les dimensions, la dynamique de formation des rides et donc la rugosité du fond. Les phénomènes de tri sédimentaire sont complexes et difficiles à estimer. Les études qui abordent ce sujet sont encore rares. On distingue deux types de tri : le tri en surface et le tri en profondeur. La plupart des études reportent que les grains les plus fins apparaissent aux creux des rides alors que les grains les plus gros sont observés aux crêtes (FOTI \& BLONDEAUX, 1995 ; DOUCETTE, 2002; ROUSSEAUX et al., 2004 ; BALASUBRAMANIAN et al., 2008). Les contraintes de cisaillement étant plus importantes aux crêtes des rides qu'aux creux, les grains les plus fins tombent dans les creux des rides, laissant les grains les plus gros aux crêtes. ROUSSEAUX et al. (2004) interprètent le tri en surface comme étant signature d'un tri en profondeur, observé grâce aux parois transparentes de leur installation.

\section{Dispositif expérimental et techniques de mesure}

\subsection{Dispositif expérimental}

Les expériences ont été menées dans un canal à houle $(10 m \times 0,5 m \times 0,5 m)$ au LOMC de l'université du Havre. Un fond sableux plat est soumis à l'action d'une houle régulière générée par un batteur et contrôlée à l'aide de sondes résistives. Le canal est muni d'une plage d'amortissement permettant ainsi de limiter au maximum la réflexion de la houle.

Les tests sont d'abord réalisés avec des sédiments bien triés de gammes granulométriques différentes. $\left(d_{50}=111 \mu \mathrm{m}, d_{50}=163 \mu \mathrm{m}, d_{50}=375 \mu \mathrm{m}\right.$ et $\left.d_{50}=175 \mu \mathrm{m}\right)$. Les conditions de houle ont été choisies telles que $2000<R_{e}=a^{2} \omega / \nu<30000$, $17<R_{d}=a \omega d_{50} / v<120$ et $3<\psi=(a \omega)^{2} /(s-1) g d_{50}<38$, où a est l'amplitude orbitale de la houle, $\omega$ la pulsation de la houle, $v$ la viscosité cinématique de l'eau et $\mathrm{s}$ la densité relative du sable. La technique expérimentale permettant le suivi spatio-temporel de la bathymétrie du fond sableux, initialement plat, est optique (MARIN \& EZERSKY, 2008). Une caméra crayon filme la trace laissée par une nappe laser sur la surface du lit, perpendiculairement au sens de propagation de la houle. L'instrumentation est fixée à un chariot qui se déplace sur un rail le long du canal. 


\subsection{Méthode d'analyse}

A partir du film enregistré par une caméra mobile, une représentation en niveaux de gris représentant la position du fond sableux sur l'ensemble de la veine d'essai est obtenue. Le domaine ainsi observé a pour dimensions $35 \mathrm{~cm}$ de large et $6 \mathrm{~m}$ de long avec une résolution égale à $0,5 \mathrm{~mm} /$ pixel dans les trois dimensions. La détection des crêtes (JARNO-DRUAUX et al., 2004) et des creux permet d'obtenir la distribution à un instant donné des longueurs d'ondes $L$, hauteurs de rides $h$ et cambrures locales $h / L$ sur l'ensemble de la veine d'essai. De plus, on considèrera un paramètre plus global de la ride : sa section $S$ (aire calculée entre deux creux de rides). L'analyse de la forme des distributions (valeur dominante, moyenne, probabilité de présence et écart type) permet d'obtenir une connaissance précise de l'état du réseau à chaque instant.

\section{Résultats}

\subsection{Croissance des caractéristiques géométriques des rides}

Chaque caractéristique géométrique $X$ de ride suit une courbe de croissance exponentielle du type : $X / X_{0}=1-\exp \left(-t / \tau_{\chi}\right)$, où $X$ représente la caractéristique de ride, $X_{0}$ sa valeur à l'équilibre et $\tau_{X}$ le temps caractéristique nécessaire à $X$ pour atteindre sa valeur finale $X_{0}$. Les vitesses de croissance diffèrent selon la grandeur considérée. L'échelle des temps dépend de $1 / \psi^{0,5}$ concernant les hauteurs et les longueurs et en $\psi / R_{e}{ }^{2}$ pour la cambrure et la section de ride. De plus, le réseau s'organise en deux étapes : au cours d'une première phase rapide ( $\sim 600$ cycles), l'organisation des rides est locale puis, le réseau se réorganise plus lentement à l'échelle globale du canal ( $\sim 600$ cycles).

\subsection{Cas avec fonds sédimentaires hétérogènes en taille}

Les essais sont effectués avec deux conditions initiales différentes de préparation du lit, d'une part une superposition de deux couches de sable $\left(90 \%\right.$ sable moyen $d_{50}=375 \mu \mathrm{m}$ et $10 \%$ sable fin $d_{50}=174 \mu \mathrm{m}$ ), et d'autre part un mélange homogène de ces sédiments. L'étude morphodynamique de l'interface a permis de mettre en évidence un ralentissement dans l'organisation des grains en surface dès les premiers instants de la formation des rides, résultat observé à l'aide de l'évolution lente de la section de ride. Une possible explication est que l'hétérogénéité en taille des grains intensifie le frottement interne, freine l'écoulement dans la couche superficielle et limite ainsi le déplacement des grains.

Le tri en surface, étudié grâce à des prélèvements, est observé plus rapidement que le tri en volume ( $\sim 4000$ cycles). Une très fine ligne de grains fins à la crête des rides.

Le tri en volume est étudié à l'aide d'une nouvelle technique expérimentale. Celle-ci vise à extraire des blocs de rides de la veine d'essai, à les injecter de résine, puis à les découper. Une analyse de ces sections de réseau de rides permettra d'étudier le tri en profondeur. Il apparait que les temps d'excitation nécessaires à l'équilibre au sein de la 
couche granulaire sont très importants ( 150000 cycles). La limite de la couche mobilisée, dont l'épaisseur varie en fonction de la position de la ride, apparait très nettement, limitée à sa base par une couche de grains fins. Sur la base des travaux de PRECHT \& HUETTEL (2004), on peut proposer une explication à ce résultat. Lors de la décompaction de la couche sableuse, les grains le plus fins auront tendance à s'infiltrer en profondeur dans les interstices laissés par les plus gros jusqu'à se trouver piégés. Ils forment la base de la couche mobilisée, pouvant ainsi jouer le rôle de filtre.

\section{Conclusions}

La croissance de réseaux de rides, formés sous l'action de la houle en canal, est caractérisée. Au cours d'une première étape, la croissance des rides est locale et rapide, puis le réseau s'organise lentement à l'échelle du canal. Les caractéristiques géométriques de rides $(L, h, h / L$ et $S)$ n'évoluent pas à la même vitesse. La section de ride, liée à l'arrangement des grains au sein de la ride, est la caractéristique qui nécessite le plus de temps pour atteindre sa valeur à l'équilibre. L'hétérogénéité en taille des sédiments dans la couche sableuse ralentit la prise de forme des rides. Le tri en volume, étudié grâce à une technique expérimentale innovante, requiert des temps plus longs que ceux nécessaires au tri en surface. La répartition des grains en profondeur est schématisée et un mécanisme lié à l'écoulement interstitiel est proposé comme première explication de cette structure. Ce type d'étude devrait permettre de mieux comprendre les mécanismes de formation des rides en ajoutant aux phénomènes hydrodynamiques, des phénomènes liés aux mouvements des grains dans la couche perméable.

\section{Références bibliographiques}

BAGNOLD R.A. (1946). Motion of waves in shallow water. Interaction between waves and sand bottoms. Proceedings of the Royal Society of London, Series A, n 187, pp 1-15.

BALASUBRAMANIAN S., VOROPAYEV S.I., FERNANDO H.J.S. (2008). Grain sorting and decay of sand ripples under oscillatory flow and turbulence. Journal of Turbulence, $\mathrm{n}^{\circ}$ 9, pp 1-19.

DOUCETTE J. (2002). Geometry and grain-size sorting of ripples on low-energy sand beaches: field observations and model predictions. Sedimentology, $\mathrm{n}^{\circ} 49$, pp 483-503.

FOTI E., BLONDEAUX P. (1995). Sea ripple formation: the heterogeneous sediment case. Coastal Engineering, $\mathrm{n}^{\circ} 25$, pp 237-253.

JARNO-DRUAUX A., BROSSARD J., MARIN F. (2004). Dynamical evolution of ripples in a wave channel. European Journal of Mechanics B/Fluids, $n^{\circ} 23$, pp 695-708.

MARIN F., EZERSKY A.B. (2008). Formation dynamics of sand bedforms under solitons and bound states of solitons in a wave flume used in resonant mode. European journal of mechanics. B, Fluids, $n^{\circ}$ 27, pp 251-267.

PRECHT E., HUETTEL M. (2004). Rapid wave-driven advective pore water exchange in a permeable coastal sediment. Journal of Sea Research, $n^{\circ}$ 51, pp 93-107.

ROUSSEAUX G., CAPS H., WESFREID J.E. (2004). Granular size segregation in underwater sand ripples. Eur. Phys. J. E, n 13, pp 213-219. 